\title{
Reply from Professor Dr Enno Freerksen
}

Let me thank you for the honouring words which I accept for my co-workers at the same time. First of all, I pass thanks to Mrs Rosenfeld with whom I have been studying these problems for decades, and furthermore to the great number of coworkers abroad, especially to those who are directly engaged in today's colloquy: Dr Alvarenga from Asunción and Dr Depasquale from Malta. Both will report today on their results.

There is no other way than giving honours always to persons. But it has to be asked what advantage patients have from our work, and where the imperfections are which directly concern practice but only can be removed by scientific work. Please, accept some short remarks in this connection.

In scientific studies on chemotherapy a lot of factors must be considered but effectivity, tolerability, practicability, and acceptance will always remain as the big four on which success depends for each therapy. If only one of these four is missing, there cannot be an effective treatment. Even high effectiveness does not help when there is no tolerance. The best therapy is also worthless when not being applied. And all is in vain when medication is ref used by the patient - indifferent by which reasons.

Although the fixed combination Isoprodian-RMP includes four drugs, the daily dosage does not exceed $2.000 \mathrm{mg}$ for adults. This is of great importance because of the compliance. The medication does not require a differentiation between multi- and pauci-bacillary cases with the consequence of saving time and money and avoiding faults. By including RMP into the fix combination, such a 
high level of safety could be reached that no more medical supervision is necessary than for any other outpatient treatment.

The application is really simple: 2 tablets in the morning and 2 in the evening - nothing could be more simple. So, we have at hand a highly effective, easily applicable and well tolerated therapy which can be used everywhere for out-patient as well as for clinical treatment.

In spite of this favourable situation, we still need new and more therapy regimens. There cannot be a uniform medication for the whole world. Any progress in science would be cancelled by this. But even independent to this point - a physician needs alternatives in order to adapt treatment to individual and regional requirements. In addition, we should have courage to expand our aims. While an effective treatment is given to as many patients as possible-with flexibility and alternatives - we must try to reach in days or weeks what nowadays takes months or even years. To search for new substances therefore will still be one of our most important tasks for the future. My personal opinion is that due to practicability, acceptability and safety fix combinations will be a great help.

But not only are more drugs needed. We also need more clearness in some basic questions regarding leprosy (i.e. methods for differentiation of viable and nonviable bacteria, significance of various types of bacteria, BI and termination of treatment) and so on.

Urgently required are clear definitions for some terms which obviously are used with quite different meanings: what means 'eradication', what is a 'relapse'? Are there any relations between leprosy reactions and chemotherapy (and if so, which)? What is the sense of 'generation time' considering the specific mode of multiplication of mycobacteria? Of course, this catalogue of questions could be completed. It seems that some discussions up to now remain unproductive because of their indistinctness of definition.

Leprosy therapy in principle was pushed forward by means of the introduction of combined therapy (MDT) and by carrying out the Malta eradication programme. Leprosy could be taken out of its isolation by transforming treatment of leprosy and tuberculosis to a 'chemotherapy of mycobacterioses'. This will be demonstrated with the great number of cases of the Paraguay project.

Today's colloquy will be an important contribution in this direction. I am really glad that it is held in Würzburg as I dare say that there is no other organization to which we owe so many thanks than to the German Leprosy Relief Association. 